\title{
Reflecting on the main findings and practical applications
}

\author{
Bart Rienties, Regine Hampel, Eileen Scanlon and \\ Denise Whitelock
}

\section{I Introduction}

The main objective of this book Open World Learning: Research, Innovation and the Challenges of High-Quality Education was to establish an informed theoretical and methodological basis for research and practical application of open world learning. As highlighted throughout this book, open world learning gives unprecedented access to information, knowledge, and education and provides support to learners across the globe. However, it is not the educational technologies themselves that represent the biggest change, but the opportunities for openness that flow from their thoughtful application, in the form of availability of and access to open and “closed" learning opportunities (Ferguson, Jones, \& Scanlon, 2019; Hampel, 2019; Nguyen, Rienties, \& Whitelock, 2022; Rizvi, Rienties, Kizilcec, \& Rogaten, 2022).

The main question of this book was: How can open world learning supported by technology help and/or hinder tackling the global challenges that open and high-quality education faces? First, this book provided several integrated and cohesive perspectives of the affordances and limitations of open world learning. The eighteen chapters brought together a range of research communities, including colleagues in artificial intelligence (Hillaire, Rienties, Fenton-O'Creevy, Zdrahal, \& Tempelaar, 2022), computing (Iniesto \& Hillaire, 2022), education (Anastasiou, 2022; Mohamud, Buckler, Pitt, \& Twining, 2022; Srisontisuk, 2022), educational psychology (Hall, Herodotou, \& Iacovides, 2022), human-computer interaction (Iniesto, McAndrew, Minocha, \& Coughlan, 2022), language education (Conde Gafaro, 2022; Vogiatzis, Charitonos, Giaxoglou, \& Lewis, 2022), learning analytics (Korir, Slade, Holmes, \& Rienties, 2022; Nguyen et al., 2022; Rizvi et al., 2022), linguistics (Chua, 2022; Rets, Stickler, Coughlan, \& Astruc, 2022), and professional learning (Chaudhari, Littlejohn, \& Cross, 2022; Iwaniec-Thompson, 2022).

Second, this book featured a wide range of open world learning topics, ranging from theoretical and methodological discussions to empirical demonstrations of how open world learning can be effectively implemented, evaluated, and used to inform theory and practice. This book provided in-depth analyses of the (potential) benefits and limitations of open world learning by bringing together insights from 387,134 learners and educators learning and working in 136 unique learning contexts across the globe (e.g., blended language learning in Italy and the UK, 
Internet kiosks in Uganda, MOOCs involving learners across the globe, online courses in the UK, science learning in Greece). This interconnected body of work not only has tremendous reach, but by comparing and contrasting different learning experiences of academics, children, educators, gamers, learners, instructional designers, managers, professionals, students, teachers, and young adults in a range of local, national and international settings, the sum of these 136 learning contexts is more than each of its individual parts.

Third, this book brought together a range of innovative uses of technology in open world learning, such as digital games (Hall et al., 2022), Futurelearn (Chua, 2022; Conde Gafaro, 2022; Iniesto et al., 2022; Rizvi et al., 2022), iPads (Srisontisuk, 2022), open educational resources (Rets et al., 2022), solar-powered Internet kiosks (Mohamud et al., 2022), (student sourced) sentiment analysis classifiers of online chat (Hillaire et al., 2022), WhatsApp (Vogiatzis et al., 2022), and Youtube (Anastasiou, 2022). A range of complex and interlinked research methodologies have been used to understand how educational technology is used by people in open world learning settings. Several innovative methodologies were used, including quantitative methodologies such as discourse analysis (Chua, 2022), eyetracking (Rets et al., 2022), learning analytics (Nguyen et al., 2022; Rizvi et al., 2022), machine learning (Hillaire et al., 2022), and (psychometric) online surveys (Conde Gafaro, 2022; Hall et al., 2022; Korir et al., 2022). Furthermore, a range of in-depth qualitative methodologies were employed throughout this book, including (ethnographic, longitudinal) observations (Anastasiou, 2022; IwaniecThompson, 2022; Mohamud et al., 2022; Srisontisuk, 2022), and thematic analysis of artefacts, documents, interviews, and other qualitative data (Anastasiou, 2022; Iniesto et al., 2022; Mohamud et al., 2022; Srisontisuk, 2022; Vogiatzis et al., 2022). Finally, several chapters specifically embraced mixed method approaches (Iniesto et al., 2022; Rets et al., 2022; Rizvi et al., 2022).

In the remainder of this chapter, we will not try to summarise each of the chapters, but rather try to place the findings into a wider context of the open world learning framework previously described in Chapter 2 (Rienties, 2022). This framework helps to depict what we have learned about what works in open world learning, what practical advice we give to learners, educators, and institutions, and finally what the next steps in open world learning research could be. As is evident throughout this book the enablers and disablers for open world learning continuously change the fluidity of the open world learning framework. This means that the size and space of open world learning experienced by individual learners and organisations in any specific context might be substantially different from other learners and organisations, and will inevitably change over time as technology, society, and people continuously interact in a flux.

\subsection{What is now known about what works in Open World Learning}

As argued in Chapter 2, while substantial progress had been made in mapping and reviewing the macro (i.e., regional, national, international, global) trends and 
concepts of open world learning until 2014, there was a paucity of evidence-based research how learners and educators make use of open world learning on a meso (i.e., institutional, cross-institutional, cross-discipline) and micro level (within institution, module, student). Chapters 14 and 15 specifically focussed on a meso level how MOOC organisations (Iniesto \& Hillaire, 2022) and course designers at the Open University (Nguyen et al., 2022) make decisions in terms of how to design effective and inclusive learning designs and experiences.

Six chapters (Chaudhari et al., 2022; Iniesto et al., 2022; Iwaniec-Thompson, 2022; Mohamud et al., 2022; Rizvi et al., 2022; Srisontisuk, 2022) combined a meso perspective with more fine-grained micro-level analyses to understand how individual learners and educators are making decisions within a broader context. For example, in Chapter 17 (Chaudhari et al., 2022) a convincing narrative is provided how experts and practitioners in the finance sector dealt with uncertainty, and how this was influenced by both individual as well as meso-factors. Similarly, in Chapter 8 Rizvi et al. (2022) showed that how learners from across the globe engage with MOOCs is influenced both by micro factors (e.g., demographics, prior knowledge, motivation) as well as meso or even macro factors (e.g., culture, national educational provision).

Equally important, seven chapters (Anastasiou, 2022; Chua, 2022; Conde Gafaro, 2022; Hall et al., 2022; Hillaire et al., 2022; Korir et al., 2022; Rets et al., 2022) specifically focussed on the lived experiences of learners and educators engaging with open world learning on a micro-level. For example, Chapter 11 (Korir et al., 2022) explored how 447 students reacted to variations in privacy risks and/or benefits interventions in an experimental design. Chapter 3 (Anastasiou, 2022) explored children who received a different sequence treatment in using digital stories in science, with the results showing that children from an early age can creatively use a range of open world learning tools to tell their own stories and make meaning in their own language.

Irrespective of whether the focus was on a meso or micro level, the two main lessons from this book are (1) technology is not neutral; (2) open world learning is not necessarily open for everyone.

\subsection{Technology is not neutral}

Throughout this book how, why and with whom learners and teachers are engaging in open world learning was substantially influenced by the affordances and limitations of a respective (educational) technology. For example, while WhatsApp is omnipresent in many people's lives, just starting a suite of language tasks on WhatsApp to learn German is not a guarantee for success (Vogiatzis et al., 2022). While tablets provide teachers and children with opportunities to gain new insights and knowledge, at the same time tablets may distract from natural play and interaction (Srisontisuk, 2022). How MOOCs are designed often favours Western learning approaches as they are designed by Western educators (Rizvi et al., 2022), thus "forcing" learners from other cultures to adopt an unfamiliar learning approach. In addition, as educators' choices in terms of which learning design activities are 
included or excluded in a particular week of learning are based upon their own experiences and expectations, this inevitably has profound implications for how learners are expected to study (Nguyen et al., 2022).

\subsubsection{Open world learning is not necessarily open for everyone}

Building on the first main lesson, even with the best Internet access, IT infrastructure, and educational support available, not everyone will benefit equally from the opportunities of open world learning. For example, Iniesto et al. (2022) showed that learners with accessibility needs often struggle to make use of open world learning. In MOOCs interactions, for example, some types and groups of learners are often more present than others (Chua, 2022; Rizvi et al., 2022), thereby potentially reducing the (heard) voices from other learners. As most open world learning opportunities are created in the medium of English, non-native English speakers are often put at a disadvantage in terms of benefitting from engagement with OERs or MOOCs for learning (Chua, 2022; Rets et al., 2022). While the community approach of Internet kiosks in Uganda by Mohamud et al. (2022) showed that learners in a community can teach each other to learn to use open world learning tools, this approach may not necessarily work in other contexts.

While the authors in this book acknowledge that technology is not neutral, and that not everyone may benefit equally from open world learning, throughout the book there are markers and evidence-based findings supported by robust methodologies how you as learner, educator, or as manager can ensure that you can benefit from the powers of open world learning. Implementing some of the practical advice may help you to become a more successful open world learner and/or educator, as illustrated in section 19.3.

\subsection{Practical advice to support and mediate effective open world learning}

\section{Empowering learners (how to become an effective open world learner)}

- Set your own goals what you want to achieve in open world learning (Chapters 5 and 9).

- Follow the learning design schedule set in open world learning, and adjust where needed (Chapter 8 and 14).

- Get involved in (co-)designing open world learning (Chapter 9 and 15).

- Think carefully about including open and inclusive language. This will increase constructive engagement with other learners (Chapter 6).

- Position yourself as a digital story maker and take the opportunity to do so in every step of the creation process in open world learning (Chapter 3).

\section{Empowering innovative technologies (how to use them)}

- There is no single universal learning design that works for all learners (Chapters 5, 6, 8, and 9).

- The mere use of innovative tools like WhatsApp or Massive Open Online Courses (MOOCs) cannot guarantee interaction and knowledge 
construction amongst your learners. These tools need to be appropriately embedded into your learning design (Chapters 4 and 6).

- As long as the linguistic accessibility of online materials is ignored, and these resources continue to draw on native speaker capital in language, the capacity of these resources to widen access to quality education will only remain that: a potential (Chapters 7 and 9).

- When using artificial intelligence tools to identify complex patterns in data (e.g., emotions, discourse), you should consider how to align the lived experience of students to model highly subjective topics (Chapters 6 and 13).

- Think about possible concerns regarding the usage of smartphones and tablets with young children (Chapter 16).

- Solar-powered Internet kiosks can support low-income communities to achieve their desired goals and facilitate soft skills development (Chapter 10).

- As a developer you could use the Creative Gaming scale as a guide on what aspects of creativity are most important to players and learners (Chapter 12).

\section{Empowering educators (what you need to do as educator)}

- It is crucial for you as educator to identify how learners set and assess their goals in open world learning (Chapter 5).

- Simplify your language! We repeat: Simplify your language! (Chapter 7).

- There are many potential misalignments between what you as an educator think your learners do and what they actually do (Chapter 14).

- As educator you should seek a better understanding of your learners and their needs (Chapter 9).

- Set clear guidelines on specific, realistic, measurable, and attainable goals so that your learners can effectively self-regulate their learning in an open world (Chapter 5).

- It is important to have frequent check-ins with your learners, not only at the point of an assignment deadline but throughout their learning process (Chapter 14).

- Simplification strategies in writing online materials, such as splitting sentences, choosing words of a shorter length and higher frequency, and using fewer nouns and more connectives between/within sentences have a beneficial effect on the text processing of learners, in particular non-native English speakers (Chapter 7).

- Use digital stories to communicate complex information (Chapter 3).

- There is a strong need for flexible, culturally adaptive learning designs of open world learning, taking a balanced approach by combining different types of learning activities (so not just more text and videos) (Chapter 8).

\section{Empowering your educators (how to get the best out of your staff and learners)}

- Any online course development processes need to be reviewed from the early design stages to produce accessible content (Chapters 9 and 15).

- Your design focus should change from meeting legislative requirements to meeting learners' needs (Chapters 9 and 15). 
- Extensive training of educators in using innovative technologies and pedagogies is needed to ensure the full potential of open world learning. So do provide more training and support if your educators or staff need them (Chapters 4 and 17).

- Institutions should examine ways to empower students with respect to the use of their data by allowing them to indicate whether and which data items they would be willing to share (Chapter 11).

- Be transparent regarding the use of student data (Chapters 11 and 13).

- There are several tensions caused by the dynamic and multiple identities of (older) academics. Becoming familiar and proficient with open world learning takes time and resource (Chapter 18).

- Organisations need to exercise transparency; if there is uncertainty, this needs to be clearly communicated in order to prevent loss of trust of their employees (Chapter 17).

\subsection{What is next for open world learning?}

As indicated in Figure 19.1, a lot of progress has been made in understanding how people make use of open world learning, both on a meso as well as a micro level. All but one Chapter specifically focussed on how learners and educators used particular open world learning approaches in their context. Some of this usage of open world learning seems to depend on individual people factors, such as accessibility needs (Iniesto et al., 2022), age (Iwaniec-Thompson, 2022; Srisontisuk, 2022), conceptions of teaching (Nguyen et al., 2022), emotions (Hillaire et al., 2022), engagement (Chua, 2022; Rizvi et al., 2022; Vogiatzis et al., 2022), language skills (Chua, 2022; Rets et al., 2022), self-regulation (Conde Gafaro, 2022), and socio-economic factors (Rizvi et al., 2022).

Furthermore, some substantial progress has been made in terms of practices, as the practices people and institutions are surrounded by influence how they engage with open world learning. For example, Chapter 9 (Iniesto et al., 2022) showed how the organisational practices around MOOC providers and the legislative context substantially influenced how accessible a particular learning unit was. Chapter 16 indicated that how children were able to use iPads in the classroom depended in part on the practice in their respective school (Srisontisuk, 2022). Overall, our 136 unique learning contexts discussed in this book provide a rich and diverse overview of some of the practices used in open world learning.

\begin{tabular}{l|ccccc|}
\multicolumn{1}{c}{} & People & Places & Practices & \multicolumn{1}{c}{ Properties } \\
\cline { 2 - 5 } Meso & 1 & 0 & 2 & 2 & 5 \\
Meso/micro & 6 & 2 & 6 & 1 & 15 \\
Micro & 8 & 1 & 1 & 4 & 14 \\
\cline { 2 - 5 } & 15 & 3 & 9 & 7
\end{tabular}

Figure 19.I Heatmap of 4P themes covered in this book. 
While in 2014 there were hardly any studies on the properties of open world learning technologies and data, substantial progress has been made in this book, with seven contributions focusing on how data use might enable or thwart open world learning. For example, Chapter 11 (Korir et al., 2022) showed that most of the 447 UK students involved in an online experiment focusing on privacy and learning analytics were reasonably comfortable to share their data with higher education institutions. Chapter 12 (Nguyen et al., 2022) showed that OU educators made substantial use of student engagement data to determine how to design online courses. At the same time, with the triangulation of more and more data and Artificial Intelligence becoming more intertwined with education (Rienties, Køhler Simonsen, \& Herodotou, 2020), there are substantial concerns about how algorithms are potentially making decisions (e.g., in- or excluding learners on a particular invention, profiling, automatic feedback) that could influence behaviour and performance of learners (Baker \& Hawn, 2021).

As evidenced by Figure 19.1, relatively few Chapters focussed on places, which is perhaps surprising as places where people learn, work and live might substantially impact on how they get access to open world learning opportunities. With governments across the globe setting national agendas for (open and closed) education, and some governments restricting access to Internet and knowledge, more research is needed how places impact on learners and learning. Beyond actively restricting content and access to open world learning, even when learning activities are openly available, this does not necessarily imply that users will universally make sense of them in the same way. For example, in Chapter 8 Rizvi et al. (2022) showed that MOOCs were not culturally inclusive, with large differences in engagement patterns by 49,582 learners in 10 Futurelearn MOOCs. Therefore, there is an urgent need to understand how the construct of places influences open world learning and the lived experiences of people who live in those places.

Given the growing maturity of open world learning research, now is the time to usefully gather, compare and contrast more data on a wide range of experiences from a micro-meso level to inform an evidence-based macro perspective of open world learning. As editors we hope that this book has been inspiring, and that you might try some of these tools, approaches, and pedagogies in your own context. Please share your perspectives and insights with us and tell us what has worked, and what has not worked via our social media (@IETatOU). Only together can we help to further empower open world learning.

\section{References}

Anastasiou, P. (2022). Digital stories in science: the role of story sequencing. In B. Rienties, R. Hampel, E. Scanlon, \& D. Whitelock (Eds.), Open world learning: research, innovation and the challenges of high-quality education (pp. 29-43). London: Routledge.

Baker, R. S., \& Hawn, A. (2021). Algorithmic bias in education. https://doi.org/10.35542/ osf.io/pbmvz.

Chaudhari, V., Littlejohn, A., \& Cross, S. (2022). Antecedents and consequences of uncertainties perceived by finance professionals. In B. Rienties, R. Hampel, E. Scanlon, \& D. Whitelock (Eds.), Open world learning: research, innovation and the challenges of high-quality education (pp. 243-249). London: Routledge. 
Chua, S. M. (2022). Discourse practices in MOOC discussions: a corpus linguistic approach. In B. Rienties, R. Hampel, E. Scanlon, \& D. Whitelock (Eds.), Open world learning: research, innovation and the challenges of high-quality education (pp. 76-88). London: Routledge.

Conde Gafaro, B. (2022). First steps towards self-regulated learning: setting goals in MOOCs. In B. Rienties, R. Hampel, E. Scanlon, \& D. Whitelock (Eds.), Open world learning: research, innovation and the challenges of high-quality education (pp. 63-75). London: Routledge.

Ferguson, R., Jones, A., \& Scanlon, E. (2019). Educational visions: the lessons from 40 years of innovation. London: Ubiquity Press.

Hall, J., Herodotou, C., \& Iacovides, I. (2022). Measuring player creativity in digital entertainment games using the Creativity in Gaming Scale. In B. Rienties, R. Hampel, E. Scanlon, \& D. Whitelock (Eds.), Open world learning: research, innovation and the challenges of high-quality education (pp. 157-170). London: Routledge.

Hampel, R. (2019). Disruptive technologies and the language classroom. Cham: Springer.

Hillaire, G., Rienties, B., Fenton-O'Creevy, M., Zdrahal, Z., \& Tempelaar, D. T. (2022). Incorporating student opinion into opinion mining: a student sourced sentiment analysis classifier. In B. Rienties, R. Hampel, E. Scanlon, \& D. Whitelock (Eds.), Open world learning: research, innovation and the challenges of high-quality education (pp. 171-186). London: Routledge.

Iniesto, F., \& Hillaire, G. (2022). UDL and its implications in MOOC accessibility evaluation. In B. Rienties, R. Hampel, E. Scanlon, \& D. Whitelock (Eds.), Open world learning: research, innovation and the challenges of high-quality education (pp. 208-224). London: Routledge.

Iniesto, F., McAndrew, P., Minocha, S., \& Coughlan, T. (2022). Accessibility in MOOCs: the stakeholders' perspectives. In B. Rienties, R. Hampel, E. Scanlon, \& D. Whitelock (Eds.), Open world learning: research, innovation and the challenges of high-quality education (pp. 119-130). London: Routledge.

Iwaniec-Thompson, G. (2022). The identity trajectories of older academics: workplace affordances and individual subjectivities. In B. Rienties, R. Hampel, E. Scanlon, \& D. Whitelock (Eds.), Open world learning: research, innovation and the challenges of high-quality education (pp. 250-263). London: Routledge.

Korir, M., Slade, S., Holmes, W., \& Rienties, B. (2022). Eliciting students' preferences for the use of their data for learning analytics: a crowdsourcing approach. In B. Rienties, R. Hampel, E. Scanlon, \& D. Whitelock (Eds.), Open world learning: research, innovation and the challenges of high-quality education (pp. 144-156). London: Routledge.

Mohamud, K., Buckler, A., Pitt, B., \& Twining, P. (2022). Internet kiosks in Uganda: a window of opportunities? In B. Rienties, R. Hampel, E. Scanlon, \& D. Whitelock (Eds.), Open world learning: research, innovation and the challenges of high-quality education (pp. 131-143). London: Routledge.

Nguyen, Q., Rienties, B., \& Whitelock, D. (2022). Informing learning design in online education using learning analytics of student engagement. In B. Rienties, R. Hampel, E. Scanlon, \& D. Whitelock (Eds.), Open world learning: research, innovation and the challenges of high-quality education (pp. 189-207). London: Routledge.

Rets, I., Stickler, U., Coughlan, T., \& Astruc, L. (2022). Simplification of open educational resources in English: exploring its effect on text processing of non-native English speakers. In B. Rienties, R. Hampel, E. Scanlon, \& D. Whitelock (Eds.), Open world learning: research, innovation and the challenges of high-quality education (pp. 89-102). London: Routledge.

Rienties, B. (2022). Powers and limitations of open world learning: experiences from the field of education. In B. Rienties, R. Hampel, E. Scanlon, \& D. Whitelock (Eds.), Open world learning: research, innovation and the challenges of high-quality education (pp. 13-26). London: Routledge. 
Rienties, B., Køhler Simonsen, H., \& Herodotou, C. (2020). Defining the boundaries between artificial intelligence in education, computer-supported collaborative learning, educational data mining, and learning analytics: a need for coherence. Frontiers in Education, 5(128). doi:10.3389/feduc.2020.00128

Rizvi, S., Rienties, B., Kizilcec, R., \& Rogaten, J. (2022). Culturally adaptive learning design - a mixed-method study of cross-cultural learning design preferences in MOOCs. In B. Rienties, R. Hampel, E. Scanlon, \& D. Whitelock (Eds.), Open world learning: research, innovation and the challenges of high-quality education (pp. 103-116). London: Routledge.

Srisontisuk, P. (2022). Practitioner's perspective on young children's use of mobile technology. In B. Rienties, R. Hampel, E. Scanlon, \& D. Whitelock (Eds.), Open world learning: research, innovation and the challenges of high-quality education (pp. 225-236). London: Routledge.

Vogiatzis, D., Charitonos, K., Giaxoglou, K., \& Lewis, T. (2022). Can WhatsApp facilitate interaction? A case study of adult language learning. In B. Rienties, R. Hampel, E. Scanlon, \& D. Whitelock (Eds.), Open world learning: research, innovation and the challenges of highquality education (pp. 44-62). London: Routledge. 